\section{Johannes-Wenner- Forschungspreis}

Ziel des Johannes-Wenner-Forschungspreises ist es, Forschungs- und Projektarbeiten von Mitgliedern der Gesellschaft für Pädiatrische Pneumologie zu unterstützen. Dadurch soll die Versorgung und Behandlung von Kindern mit Lungenerkrankungen verbessert werden. Der Johannes-Wenner-Forschungspreis richtet sich an Ärzte sowie Wissenschaftler, die Themen der pädiatrischen Pneumologie sowohl im klinischen Bereich wie auch im Grundlagenbereich bearbeiten möchten. Der Preis wird von der Deutschen Lungenstiftung e.V. gestiftet und ist mit $15000 €$ dotiert. Er wird an eine Person oder ein Team vergeben. Das Preisgeld soll der Bearbeitung des beantragten Forschungsprojektes dienen. Der Preis richtet sich bevorzugt an junge Mediziner und Wissenschaftler, ist aber auch für erfahrene Antragsteller offen.

Die Preisverleihung findet auf der Jahrestagung der Gesellschaft für Pädiatrische Pneumologie statt. Bewerbungsfrist endet am 10. Dezember 2013. Die Bewerbung muss von einem hauptverantwortlichen Antragsteller eingereicht werden. Lebenslauf und Publikationsverzeichnis der Antragsteller müssen beigefügt werden. Das Forschungsprojekt, für das die Förderung vorgesehen ist, soll in einer 4-seitigen Projektskizze zusammengefasst werden. Diese sollte folgende Punkte beinhalten:

- Zusammenfassung des Forschungsvorhabens (max. $12 / 2$ Seite),

- Stand der Forschung und eigene Vorarbeiten (max. 1 Seite),

- Ziele und Arbeitsprogramm (max. 1,5 Seiten),

- beantragte Mittel mit Verwendungsnachweis (max. $1 / 2$ Seite) und

- die Voraussetzungen für die Durchführung des Vorhabens (max. 1/2 Seite).

Bewerbungen sind als PDF-Datei zu senden an folgende E-Mail-Adresse: Hansen. Office@mh-hannover.de oder in schriftlicher Form an Prof. Gesine Hansen, MHH, Klinik für Pädiatrische Pneumologie, Allergologie und Neonatologie, Carl-Neuberg-Straße 1 in 30625 Hannover.

Nach einer Mitteilung der Deutschen Lungenstiftung e.V.

\title{
Welchen Nutzen hat eine ECMO bei schwerem ARDS?
}

\author{
Während der letzten Pandemie mit dem A/H1N1-Influenzavirus \\ wurden viele Patienten wegen eines schweren akuten \\ Atemnotsyndrome (ARDS) mit der extrakorporalen \\ Membranoxygenierung (ECMO) behandelt. Über den Nutzen \\ dieser Maßnahme wird kontrovers diskutiert, zumal die \\ Ergebnisse in unterschiedlichen Ländern heterogen sind. Hierzu \\ haben T. Pham et al. nun französische Registerdaten verglichen. \\ Am J Respir Crit Care Med 2013; 187: 276-285
}

Für die Auswertung wurden prospektiv Daten von Patienten mit PCR-bestätigten A/H1N1-Infektionen erhoben, die zwischen Juli 2009 und März 2011 wegen ARDS auf eine Intensivstation kamen und dort entweder mit oder ohne ECMO behandelt wurden. Zusätzlich zu den üblichen Kriterien diente ein Lung Injury Score $>3$ zur Definition des schweren ARDS. Die Vergleichsgruppenanalyse bestand in einem Propensity Score Matching von Patienten, deren ECMO-Behandlung innerhalb der 1 . Woche eines ARDS begann, mit nicht ECMO-behandelten Patienten.

In die Gesamtauswertung flossen Daten von 123 Patienten (50\% Männer) mit einem Durchschnittsalter von 42 Jahren ein. Davon erfüllten $76 \%$ einen oder mehrere Risikofaktoren für influenzabedingte Komplikationen, hauptsächlich Adipositas, Schwangerschaft, Immunsuppression oder postpartaler Status. Bei 23\% der Patienten lag eine begleitende bakterielle Infektion vor. Alle ECMO-Patienten bekamen antivirale Medikamente, und in 76\% der Fälle waren Notfallmaßnahmen nötig, überwiegend StickstoffmonoxidInhalation und zu 45\% Bauchlage. Den Gasaustausch erhielten 13\% der Patienten als venoarterielle und alle anderen als venovenöse ECMO. Die Dauer der ECMO betrug im Median 11 Tage, die Intubation 28 und die Zeit auf der Intensivstation 33 Tage. Insgesamt starben auf den Intensivstationen 36\% der Patienten. Diese Todesfälle waren nach multivariater Analyse mit dem Alter, höheren Plateaudrücken und Serumlaktatwerten assoziiert ( $\mathrm{p}$ jeweils $<0,01$ ).

\section{Keine Überlebensverbesserung mit ECMO \\ $\nabla$}

Die Daten von 103 Patienten mit ECMOBehandlung innerhalb der 1. Woche eines ARDS wurden mit denen von $157 \mathrm{~Pa}-$ tienten mit schwerem ARDS, aber ohne ECMO-Therapie verglichen. Die ECMOGruppe war im Durchschnitt jünger, der Anteil von Schwangeren und Adipösen war höher, dagegen waren Komorbiditäten, Immunsuppressionen, BakterienInfektionen bei der Aufnahme und frühe Steroidbehandlungen geringer. Die Mortalität auf der Intensivstation war in den Gruppen mit und ohne ECMO vergleichbar (36 vs. 34\%). Für die strikte Vergleichsgruppen-Analyse konnten $52 \mathrm{~Pa}-$ tienten-Paare gebildet werden. Auch hier ergab sich kein signifikanter Unterschied bei der Mortalität auf den Intensivstationen: $50 \%$ mit vs. $40 \%$ ohne ECMO-Behandlung, $p=0,32$ ).

\section{Fazit}

In dieser Studie konnte mit einer Vergleichsgruppen-Analyse kein Überlebensvorteil der ECMO für A/H1N1-infizierte Patienten mit schwerem ARDS nachgewiesen werden. Die Autoren vermuten, dass eine ultraprotektive Beatmungsstrategie mit Plateaudrücken um $25 \mathrm{~cm} \mathrm{H}_{2} \mathrm{O}$ vom 1 . Tag einer ECMO an nötig sei, um das Überleben zu verbessern.

Matthias Manych, Berlin 